\title{
A Technical Note on Quantum Dots for Multi-Color Fluorescence in situ Hybridization
}

\author{
S. Müller ${ }^{a} \quad$ M. Cremer ${ }^{a} \quad$ M. Neusser ${ }^{a} \quad$ F. Grasser ${ }^{a} \quad$ T. Cremer ${ }^{a}$ b \\ ${ }^{a}$ Department Biology II, Human Genetics, and ${ }^{b}$ Center for Integrated Protein Science Munich (CIPSM), \\ Ludwig-Maximilians University, Munich, Germany
}

\section{Key Words}

4Pi microscopy - Chromosome - Multicolor FISH • Nucleus • Nuclear architecture $\cdot$ Quantum dot

\begin{abstract}
Quantum dots (Qdots) are semiconductor nanocrystals, which are photo-stable, show bright fluorescence with narrow, symmetric emission spectra and are available in multiple resolvable colors. We established a FISH protocol for the simultaneous visualization of up to 6 different DNA probes differentially labeled with Qdots and with conventional organic fluorochromes. Using a Leica SP5 laser scanning confocal microscope for image capture, we tested various combinations of hapten-labeled probes detected with streptavidin-Qdot525, sheep anti-digoxigenin-Qdot605, rat antidinitrophenyl-Qdot655 and goat anti-mouse-Qdot655, respectively, together with FITC-dUTP-, Cy3-dUTP- and Texas Red-dUTP-labeled probes. We further demonstrate that Qdots are suitable for imaging of FISH probes using 4Pi microscopy, which promises to push the resolution limits of light microscopy to 100 nanometers or less when applying a deconvolution algorithm, but requires the use of highly photo-stable fluors.

Copyright $\odot 2009$ S. Karger AG, Basel
\end{abstract}

Quantum dots (Qdots) represent a distinct class of chemo-luminescent molecules characterized by a number of unique optical properties [Murray et al., 1993; Bruchez et al., 1998; Chan and Nie, 1998]. These crystalline semiconductor nanoparticles, typically $2-8 \mathrm{~nm}$ in size, have very similar, broad excitation spectra with a maximum at $405 \mathrm{~nm}$ and can thus be excited at a single wavelength. Depending on the respective size of a quantum dot, their emission spectra range from 500 to $800 \mathrm{~nm}$ [Bruchez, 2005 for review]. Due to their narrow and symmetric emission spectra they can be used in multi-color approaches, to visualize several targets in a single experiment [Chan et al., 2002 for review]. Moreover, Qdots have higher photo-stability compared to organic fluorophores [Wu et al., 2003]. During the last decade, these properties have raised considerable interest in using Qdots as fluorescent markers in a broad range of biological applications, including immuno-histochemistry, cell tracking, FRET, in vivo imaging, flow cytometry, and also fluorescence in situ hybridization (FISH) [Watson et al., 2003; Alivisatos et al., 2005; Medintz et al., 2005; Bruchez and Hotz, 2007; for recent reviews].

It had already been recognized during early stages of the development of Qdots for bio-medical research and

\section{KARGER}

Fax +41613061234

E-Mail karger@karger.ch

www.karger.com
(C) 2009 S. Karger AG, Basel

$1424-8581 / 09 / 1244-0351 \$ 26.00 / 0$

Accessible online at:

www.karger.com/cgr
Stefan Müller

Department Biology II, Anthropology and Human Genetics Ludwig-Maximilians-University, Grosshaderner Strasse 2 DE-82152 Planegg-Martinsried (Germany), Tel. +49 89218074325

Fax +4989218074 331, E-Mail s.mueller@lrz.uni-muenchen.de 
diagnostic purposes that the visualization of DNA probes on metaphase chromosomes and in interphase nuclei by FISH would be an important cytogenetic and cell biological application of Qdots. Their use in FISH experiments has indeed been investigated for several years [Pathak et al., 2001; Xiao and Barker, 2004]; however, surprisingly few reports on the successful application of Qdots can be found in the literature, and only recently Qdot-conjugated antibodies against haptens (for example digoxigenin or dinitrophenyl) or against biotin have become commercially available. Until today, successful visualization of FISH probes using Qdots has been reported for repetitive sequences [Pathak et al., 2001; Wu et al., 2006; Ma et al., 2008], for gene-specific probes [Xiao and Barker, 2004; Jiang et al., 2007] and for oligonucleotide probes [Byers et al., 2007; Knoll, 2007], but so far not for the delineation of entire chromosome territories by chromosome painting.

Because of their large Stoke's shift ranging between $100 \mathrm{~nm}$ and over $300 \mathrm{~nm}$, Qdots have drastically different spectral properties compared to most organic fluors, even for molecules with similar emission spectra. We therefore reasoned that Qdots are potentially useful to expand the number of fluorochromes, which can be simultaneously used in multiplex FISH experiments when combining Qdot and organic fluor-labeled DNA probes. Further, their extreme photo-stability would meet the requirements for high-resolution 4Pi laser scanning confocal microscopy of FISH probes in investigating 3-dimensional nuclear genome organization. Here we present a FISH protocol that allows for the simultaneous visualization of at least 6 different DNA probes labeled with a combination of Qdots and organic fluorochromes. Further, we provide initial results on 4Pi microscopy of chromosome territories using this methodology. Finally, we discuss current limitations and future prospects of this approach.

\section{Material and Methods}

\section{Cell Material and Fixation}

Karyotypically normal human lymphoblastoid cells or fibroblasts used in these experiments are the same as described before [Bolzer et al., 2005; Neusser et al., 2007]. Metaphase preparation from lymphoblastoid cells followed standard procedures. Morphologically preserved interphase nuclei from human fibroblast cells were prepared according to Cremer et al. [2007]. Briefly, fibroblasts were fixed in $4 \%$ paraformaldehyde, $1 \times$ PBS, followed by sequential permeabilization steps including treatment in $0.5 \%$ Triton X-100, 1× PBS; 20\% glycerol, 1× PBS; repeated freezing/ thawing in liquid nitrogen, incubation in $0.1 \mathrm{~N} \mathrm{HCl}$ and pepsin- ization $\left(2 \mathrm{mg} / \mathrm{ml}\right.$ pepsin in $0.01 \mathrm{~N} \mathrm{HCl}$ at $\left.37^{\circ} \mathrm{C}\right)$. For $4 \mathrm{Pi}$ confocal microscopy cells were grown on round glass coverslips fitting to the mounting device attached to this microscope (www.leica-microsystems.com).

\section{DNA Probe Labeling and Hybridization in situ and}

Post-Hybridization Washes

Human chromosome 2, 3, 5, 6, 7, 13, 15, 17 and 20 specific painting probes were amplified and labeled by DOP-PCR as described [Müller et al., 2007]. Depending on the respective FISH experiment, one and up to 6 different painting probes were labeled with either biotin-dUTP (BIO, Roche), digoxigenin-dUTP (DIG, Roche), dinitrophenyl-dUTP (DNP, Molecular Probes), FITC-dUTP (Molecular Probes), Cy3-dUTP (GE Healthcare) or Texas Red-dUTP (Molecular Probes). In situ hybridization was performed according to standard procedures [Schwarzacher and Heslop-Harrison, 2000, with minor modifications; Cremer et al., 2007], post-hybridization stringency washes included 3 times $5 \mathrm{~min}$ in $0.1 \times \mathrm{SSC}$ at $60^{\circ} \mathrm{C}$.

\section{Antibody Detection, Counterstaining and Embedding}

In control FISH experiments using only conventional organic fluorochromes, post-hybridization washes were followed by a 20 min blocking step in $4 \times$ SSC, $0.5 \%$ Tween $20,3 \%$ bovine serum albumin. BIO-labeled paint probes were then detected with avidin-Alexa488 (Molecular Probes) or avidin-Cy5 (Dianova). DNP-labeled probes were visualized by sequential detection with rabbit anti-DNP and goat anti-rabbit-Alexa488 (Sigma), goat anti-rabbit-Alexa514 (Molecular Probes) or goat anti-rabbit-RhodamineX (Invitrogen) antibodies, respectively. DIG-labeled probes were detected with mouse anti-DIG-Cy5 (Dianova). All antibodies were diluted according to the manufacturer's recommendation. After each antibody incubation step, slides were washed 3 times for $5 \mathrm{~min}$ in $4 \times \mathrm{SSC}, 0.5 \%$ Tween $20\left(42^{\circ} \mathrm{C}\right)$. Finally, metaphase chromosomes and $3 \mathrm{D}$ fixed-interphase nuclei were counterstained for $10 \mathrm{~min}$ with DAPI $(2 \mu \mathrm{g} / \mathrm{ml})$ and were mounted in Vectashield antifade solution.

When detecting paint probes with Qdot conjugates, the following modifications of the standard detection scheme were made. After the post-hybridization washes, the slides were equilibrated by incubation 2 times for 5 min each in $1 \times$ PBS, $0.5 \%$ Tween 20 at room temperature. BIO-labeled probes were then detected with streptavidin-Qdot525 (Tebu-bio) and DNP-labeled probes with rat-anti-DNP-Qdot655 (Tebu-bio). DIG-labeled probes were detected by sheep anti-Dig-Qdot605 (Tebubio), or sequentially, using first a mouse anti-DIG and then a goat anti-mouse-Qdot655 (Invitrogen) antibody. All Qdot conjugate stock solutions were diluted 1:50 in $1 \times$ PBS, $0.2 \%$ Tween 20 . After each antibody incubation step, slides were washed once for $5 \mathrm{~min}$ in $1 \times \mathrm{PBS}, 0.5 \%$ Tween $20\left(42^{\circ} \mathrm{C}\right)$. Slides were mounted in polyvinylalcohol mounting medium containing DABCO (Fluka), or in 97\% 2,2'-thiodiethanol (TDE) [Staudt et al., 2007]. Embedding in TDE was performed in an ascending series, including 10\% TDE (100 $\left.\mu \mathrm{l} \mathrm{TDE,} 50 \mu \mathrm{l} 5 \times \mathrm{PBS}, 850 \mu \mathrm{l} \mathrm{H}_{2} \mathrm{O}\right), 25 \%$ TDE $\left(250 \mu \mathrm{l}\right.$ TDE, $\left.50 \mu \mathrm{l} 5 \times \mathrm{PBS}, 700 \mu \mathrm{l} \mathrm{H} \mathrm{H}_{2} \mathrm{O}\right), 50 \%$ TDE $(500$ $\mu \mathrm{l}$ TDE, $50 \mu \mathrm{l} 5 \times \mathrm{PBS}, 400 \mu \mathrm{l} \mathrm{H}_{2} \mathrm{O}$ ) and 3 times in $97 \% \mathrm{TDE}$ (970 $\mu \mathrm{l}$ TDE and $30 \mu \mathrm{l} 1 \times \mathrm{PBS}$ ) for $10 \mathrm{~min}$ each [Staudt et al., 2007]. 
Conventional and 4Pi Confocal Microscopy, Image Processing, Analysis and 3D-Reconstruction

For the simultaneous visualization of up to 6 fluorochromes, images from hybridized metaphases and nuclei were recorded using a TCS SP5 AOBS laser scanning confocal microscope (Leica Microsystems) equipped with 5 laser lines (405, 488, 561, 594, 633 $\mathrm{nm})$ and a $63 \times, 1.4$ plan-apochromat oil objective. For $4 \mathrm{Pi}$ imaging of FISH experiments with RhodamineX- or Qdot655-labeled probes, a TCS 4 Pi confocal microscope (Leica Microsystems) was used in type A 2-photon excitation mode. Appropriate laser excitation lines were chosen and acusto-optical beam splitters were tuned according to standard procedures for DAPI, Alexa488/ FITC, Cy3, RhodamineX, Texas Red and Cy5 (table 1). Qdot labels were excited using the 405-nm UV diode laser line, and emission windows were chosen $\pm 25 \mathrm{~nm}$ of the respective emission maximum (table 1). Metaphases and nuclei were scanned with an axial distance of $200 \mathrm{~nm}$ (SP5) or $90 \mathrm{~nm}$ (4Pi) between consecutive light optical sections yielding separate stacks of 8-bit grayscale images for each fluorescence channel with a pixel size of $40-120 \mathrm{~nm}$. Image stacks were processed and analyzed with ImageJ software (http://rsb.info.nih.gov/ij). For quantification of the probe hybridization/detection efficiency, fluorescence intensities were measured in unprocessed FISH images using ImageJ software. At least 20 metaphase chromosome regions per experiment showing hybridization by a given paint probe were quantitatively analyzed. The level of unspecific noise was determined by fluorescence intensity measurement in 20 or more chromosome regions from the same experiment, which were not targeted by the respective paint probe (fig. 1). Deconvolution of $4 \mathrm{Pi}$ image stacks was performed using the TCS 4Pi microscope software (Leica Microsystems). 3D-reconstructions were made using Amira 3.1.1 (Mercury CS).

\section{Results and Discussion}

Here we report on the establishment of a multi-color FISH protocol that allows for the simultaneous visualization of DNA probes labeled with organic fluorochromes and with Qdot conjugates. We have successfully applied this procedure in hybridization experiments of chromosome-specific paint probes to methanol:acetic acid (MAA)-fixed metaphase chromosomes (fig. 1) and to paraformaldehyde (pFA)-fixed, 3D-preserved interphase nuclei (figs. 2-4). For image acquisition, we routinely used a conventional Leica TCS SP5 laser scanning confocal microscope. In selected experiments, hybridized nuclei were visualized on a Leica TCS 4 Pi laser scanning confocal microscope (figs. 3 and 4).

\section{Qdots Require Modifications of the Standard Probe \\ Detection Procedure}

Using the standard FISH protocol for organic fluorochrome-labeled probes established in our laboratory [Cremer et al., 2007] and the published literature on the
Table 1. Excitation and emission wavelengths used to visualize conventional fluor- and Qdot-labeled probes by the TCS SP5 AOBS laser scanning confocal microscope (AOBS = acusto-optical beam splitter)

\begin{tabular}{lll}
\hline Fluorochrome & Excitation (laser line) & Emission (AOBS) \\
\hline FITC & $488 \mathrm{~nm}$ & $525 \pm 25 \mathrm{~nm}$ \\
Cy3 & $561 \mathrm{~nm}$ & $580 \pm 10 \mathrm{~nm}$ \\
TexRed & $594 \mathrm{~nm}$ & $625 \pm 25 \mathrm{~nm}$ \\
Qdot525 & $405 \mathrm{~nm}$ & $525 \pm 25 \mathrm{~nm}$ \\
Qdot605 & $405 \mathrm{~nm}$ & $605 \pm 25 \mathrm{~nm}$ \\
Qdot655 & $405 \mathrm{~nm}$ & $655 \pm 25 \mathrm{~nm}$ \\
\hline
\end{tabular}

use of Qdots in FISH (see Introduction) as a starting point, we systematically tested the parameters needing adjustment to meet the specific requirements for both types of fluors, in order to apply them simultaneously in one experiment. Since we exclusively used Qdot-conjugated streptavidin or antibodies to detect hapten or biotin-labeled probes, we focused our attention on detection, counterstaining and embedding procedures, while probe labeling, hybridization in situ and post-hybridization stringency washes always followed standard procedures.

We reproducibly obtained optimal results with the tested Qdot conjugates when the following modifications to the standard FISH detection procedure were made. Firstly, blocking of unspecific antibody binding sites in $4 \times$ SSC, $0.5 \%$ Tween $20,3 \%$ bovine serum albumin resulted in very low signal specificity or absent fluorescence signal, and was therefore omitted. Secondly, dilution of Qdot conjugate stock solutions in high-salt $4 \times$ SSC, Tween $20,1 \%$ bovine serum albumin and $4 \times$ SSC, Tween 20 washes after antibody incubation would frequently cause precipitation of Qdot conjugates on the objective slide and absence of specific fluorescence on the targeted chromosome. Instead we performed low-salt washes in $1 \times$ PBS, Tween 20, for which we also reduced the time to a minimum required to obtain acceptable signal/noise ratios. Thirdly, when combining organic fluorophores and Qdots, Vectashield antifading solution was replaced by a polyvinylalcohol-based mounting medium containing DABCO or by TDE, respectively, for $4 \mathrm{Pi}$ confocal microscopy (see below), because the application of Vectashield caused dissolution of Qdot conjugates within minutes.

Despite these modifications, the overall performance of the Qdot conjugates tested varied considerably in our hands. Even in parallel experiments performed with identical batch-labeled paint probes, metaphase prepara- 

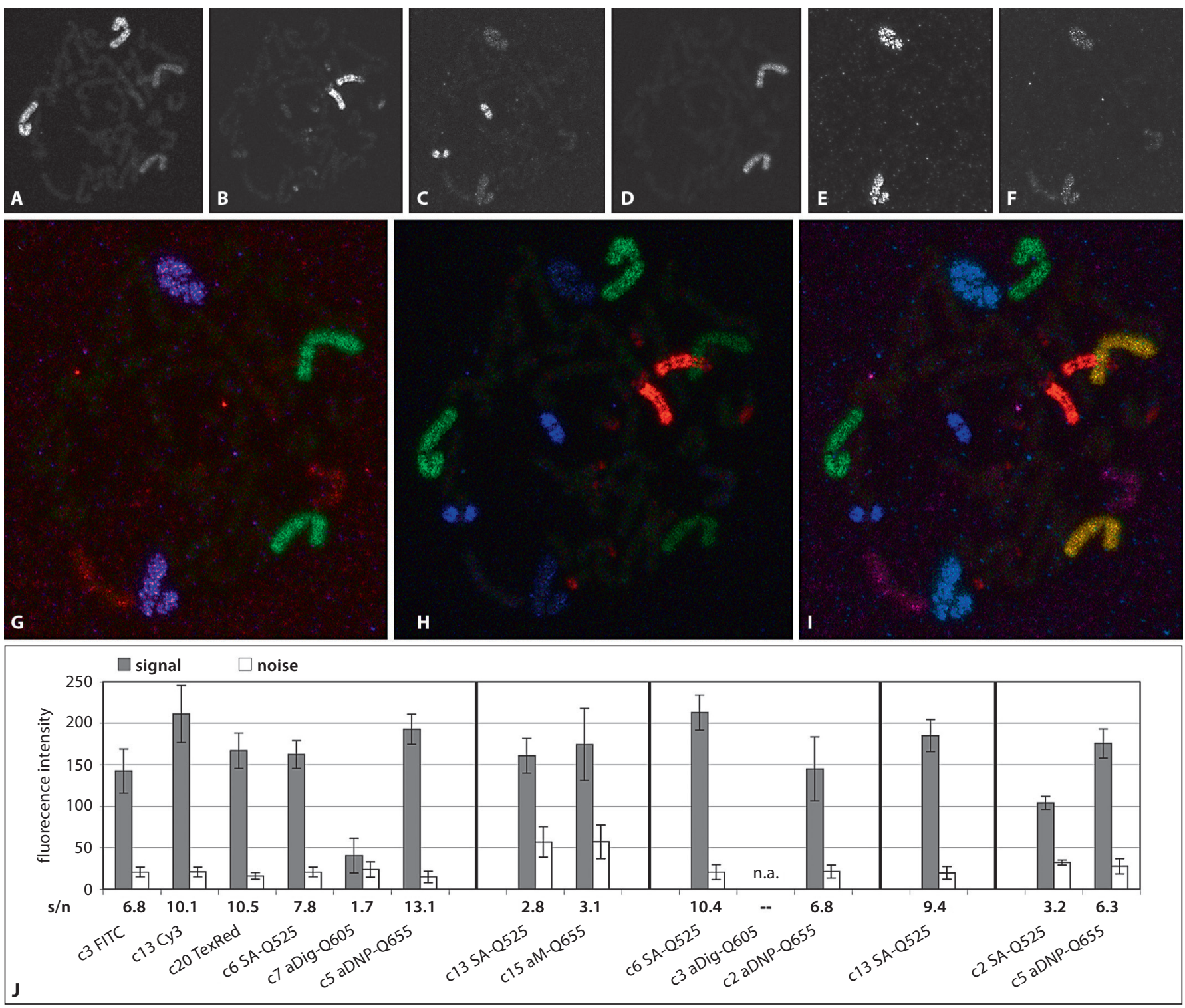

Fig. 1. Human metaphase chromosomes after multicolor FISH with 6 chromosome paint probes labeled with Qdot conjugates and organic fluorochromes. A-F Raw grayscale images. A FITC chromosome 3, B Cy3 chromosome 13, C TexasRed chromosome 20, D Qdot525 chromosome 6, E Qdot655 chromosome 5 and F Qdot605 chromosome 7. G-I RGB overlays. G Qdot525 in green, Qdot605 in red and Qdot655 in blue, H FITC in green, Cy3 in red and TexasRed in blue, I RGB overlay of all 6 probes (see text for details on the efficiency of the individual labels). J Mean fluorescence signal intensities produced by organic fluor- and Qdot-labeled paint probes, unspecific noise levels and signal/noise ratios $(\mathrm{s} / \mathrm{n})$ obtained from 5 selected metaphase FISH experiments.

tions and pre-treated slides, we frequently encountered significant differences in signal intensities and signal/ noise ratios for the same Qdot conjugate.

Overall, streptavidin-Qdot525 and anti-mouseQdot655 antibody performed best. For these two Qdot conjugates the measured mean signal intensities were comparable to those observed for conventional fluors, and good to excellent signal/noise ratios $(\mathrm{s} / \mathrm{n}=5-10)$ were frequently recorded (fig. 1J). In $20-30 \%$ of the experiments we failed to visualize paint probes using these two conjugates. For anti-dinitrophenyl-Qdot655, signal/ noise ratios of up to 13.1 could be obtained; however, the 

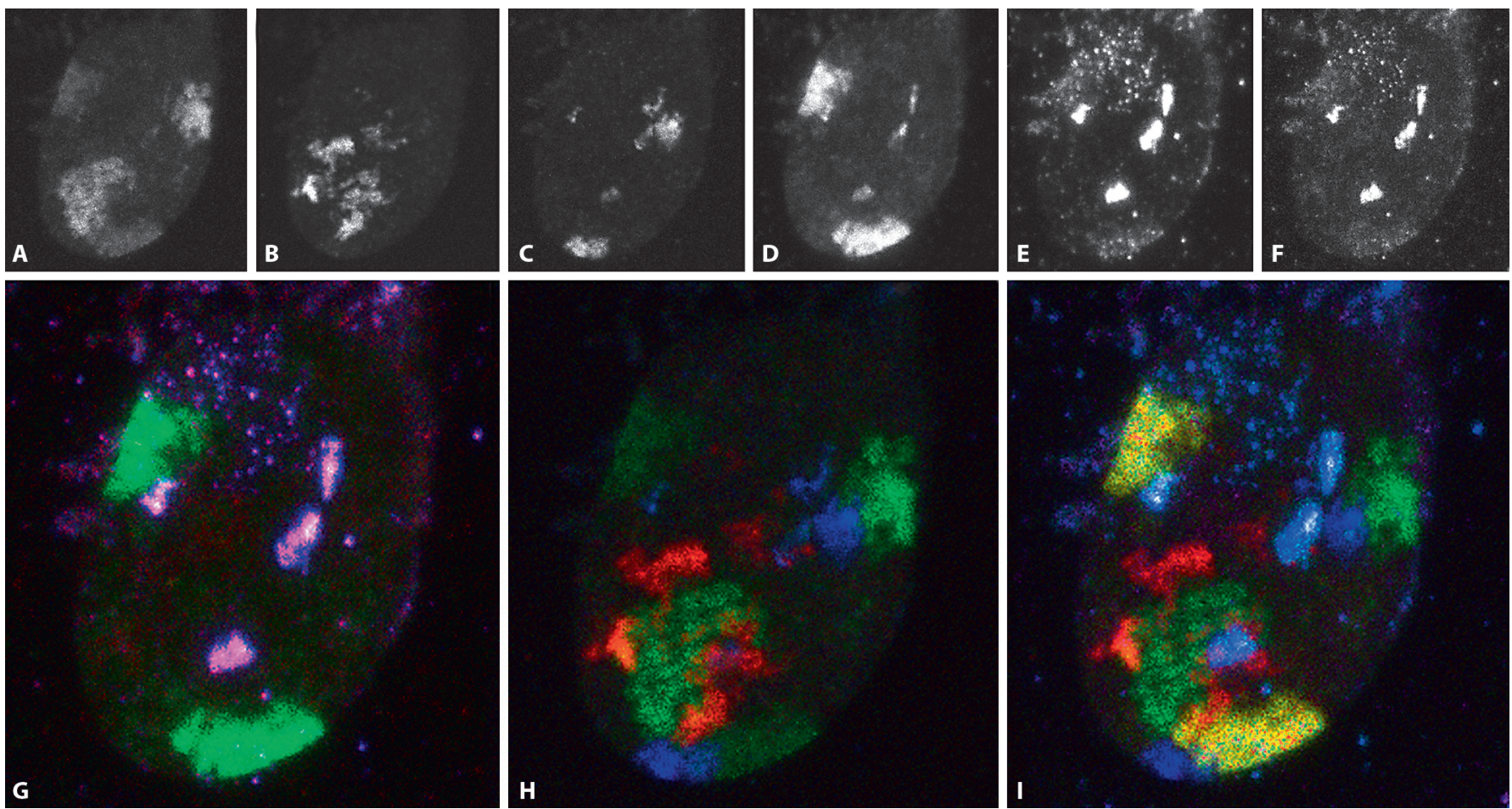

Fig. 2. Morphologically preserved human fibroblast interphase nucleus after multi-color 3D-FISH with 6 chromosome paint probes labeled with Qdot conjugates and organic fluorochromes. A-F Raw grayscale images. A FITC chromosome 3, B Cy3 chromosome 13, C TexasRed chromosome 20, D Qdot525 chromosome 6, E Qdot655 chromosome 5 and F Qdot605 chromosome 7. G-I RGB overlays: G Qdot525 in green, Qdot605 in red and Qdot655 in blue, $\mathbf{H}$ FITC in green, Cy3 in red and TexasRed in blue, I RGB overlay of all 6 probes. See text for details on the efficiency of the individual labels (note: bright, yet unspecific fluorescent spots seen in $\mathbf{E}$ and $\mathbf{F}$ originated from the anti-dinitrophenyl-Qdot655 antibody).

fluorescent pattern was typically rather coarse (fig. 1E), thus preventing detection of subtle rearrangements, and the failure rate was higher (approx. 50\%). With over $80 \%$ failure rate the anti-digoxigenin-Qdot605 antibody showed the lowest overall efficiency among the conjugates tested, and an unacceptably low signal/noise ratio when a FISH signal could be observed (for example $\mathrm{s} / \mathrm{n}=$ 1.7, fig. 1J).

The differences observed are most likely not attributed to increased Qdot size, being positively correlated with emission wavelength. Further, 2 different batches of the anti-digoxigenin-Qdot605 conjugate tested showed equally low efficiency, arguing against batch variability in the manufacturing process, and suggesting instead that not all Qdot conjugates are equally compatible with the procedure presented here. In contrast, and in agreement with previous reports [Wu et al., 2003], all Qdots that were used here successfully showed extremely stable fluorescence.

Quantum Dots for FISH
For Qdot conjugates we also noticed higher probe detection efficiency after FISH to methanol:acetic acidfixed metaphase chromosomes (fig. 1) compared to FISH on paraformaldehyde-fixed, morphologically preserved interphase nuclei (fig. 2). While paint probes directly labeled with FITC, Cy3 and Texas Red (figs. 1A-C and 2AC), as well as streptavidin-Qdot525 (figs. 1D and 2D), and the anti-mouse-Qdot655 antibody (fig. 3) reproducibly yielded satisfactory results in both instances, application of the anti-dinitrophenyl-Qdot655 antibody was restricted to methanol:acetic acid-fixed specimens (figs. $1 \mathrm{E}$ and $2 \mathrm{E})$. We assume that the restrained permeability of paraformaldehyde-fixed cells prevented the anti-dinitrophenyl-Qdot655 antibody from reaching its nuclear target. On the other hand, we can exclude that the anti-dinitrophenyl antibody component is responsible, since detection of DNP-labeled probes using a rabbit-anti-dinitrophenyl antibody is part of our standard multicolor 3DFISH detection scheme [Müller et al., 2007]. 


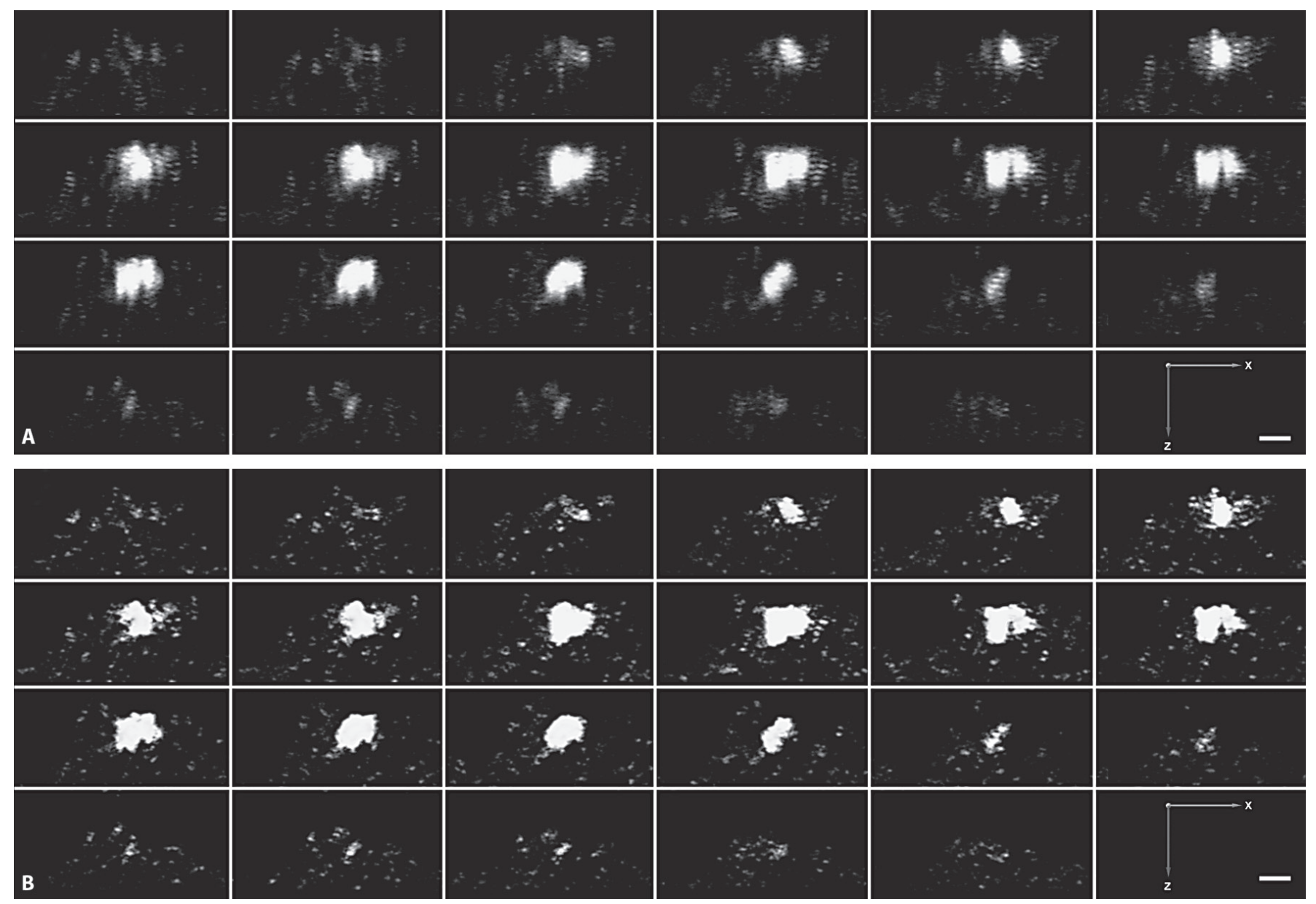

Fig. 3. Consecutive 90-nm serial $4 \mathrm{Pi}$ confocal sections of a RhodamineX-labeled chromosome 17 territory, before $(\mathbf{A})$ and after $(\mathbf{B})$ deconvolution (scale bar $=1 \mu \mathrm{m})$. Note: sections are shown in lateral view XZ.

Spectral Discrimination of Qdots and Organic

\section{Fluorochromes}

Quantum dots and conventional fluorochromes complement each other concerning their excitation and emission spectra, with small Stokes shifts being typical for most organic fluors and extremely large Stokes shifts observed for Qdots. Hence, in principle it should be possible to implement a combinatorial set of excitation and emission wavelength windows for the discrimination of Qdots and organic fluors with identical emission maxima through their Stokes shift differences. For example, DAPI, FITC and Qdot525 may be discriminated using binary combinations of exciter and emitter filters: a DAPI exciter/emitter for DAPI, a DAPI exciter/FITC emitter for Qdot525 and an FITC exciter/FITC emitter for FITC. Employing this strategy, a conventional epifluorescence microscope equipped with band pass filter sets for DAPI,
FITC, Cy 3 and Cy 5 could be used to discriminate 8 different fluorochromes (4 organic fluors and 4 Qdots) instead of only 4 , provided that excitation filters and beamsplitters/emitter filter sets would be housed in 2 separate filter wheels.

We applied this principle taking advantage of the Leica SP5 TCS laser scanning confocal microscope. Here, 5 different laser excitation channels are available, which can be combined with freely tunable emission bands. This principle worked well for the Qdot605 and the Qdot655 with longer emission wavelengths, which could be discriminated $>80 \%$ from $\mathrm{Cy} 3$ and Texas Red, respectively (figs. 1 and 2). Qdot525 could be equally well discriminated from FITC (figs. 1 and 2); however, DAPI and Qdot525 could not be spectrally separated using this microscopic setup. We expect that this issue can be resolved when replacing DAPI by an alternative counterstain with 

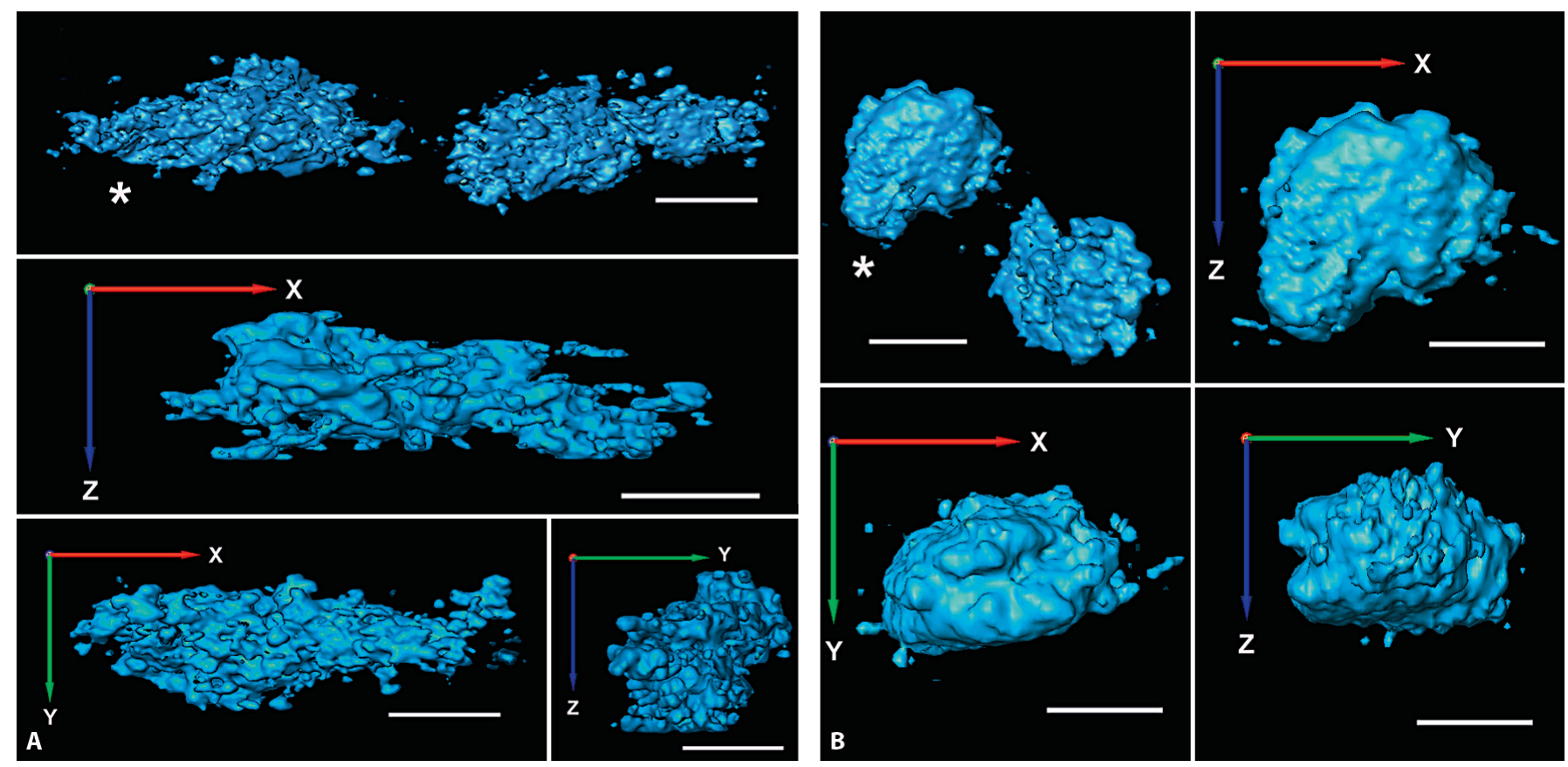

Fig. 4. 3D-reconstruction of representative chr. 17 territories labeled with Qdot655 (A) and RhodamineX (B) (asterisks mark the territory shown enlarged in XZ, YZ and XY directions; scale bar $=1 \mu \mathrm{m}$ ).

longer wavelength excitation/emission maximum, e.g. SybrGreen or TOPRO-3 (Molecular Probes), but have not tested this experimentally yet.

\section{Visualization of Chromosome Territories by $4 \mathrm{Pi}$ \\ Confocal Microscopy}

The laser scanning confocal microscope is an indispensable tool for the visualization of three-dimensional biological objects, and is therefore also widely used in studies of nuclear genome organization, for example by 3D-FISH. Conventional confocal microscopes, however, have only a limited resolution of $500-800 \mathrm{~nm}$ along the $\mathrm{z}$-axis, compared to $200 \mathrm{~nm}$ in XY. The introduction of 4Pi confocal microscopy [Hell and Stelzer, 1992] overcame this limitation, and resulted in an up to sevenfold improvement of the axial resolution. When combined with deconvolution, current 4Pi microscope technology allows for distinguishing 2 distinct objects at a distance of approximately $100 \mathrm{~nm}$ along the optical axis [Egner and Hell, 2005 for review]. Since this method is based on 2-photon excitation at 750-900 $\mathrm{nm}$ and is employing 2 opposing lenses, which create a standing wave front, fluorochromes suitable for $4 \mathrm{Pi}$ microscopy need to exhibit certain spectral characteristics and must be very photostable. In addition, the refractory index of the embedding medium should match the numerical aperture of the objective lenses to optimize the resolution [Egner and Hell, 2005], as e.g. achieved by embedding in 97\% TDE when applying a $63 \times, 1.4$ plan-apochromat oil objective.

Here we present initial successful attempts visualizing chromosome territories after 3D-FISH by $4 \mathrm{Pi} \mathrm{mi}-$ croscopy (figs. 3 and 4). For these experiments, we detected a human chromosome 17 paint probe with RhodamineX or anti-mouse-Qdot655. Both fluorochromes proved to be sufficiently bright and photo-stable to be visualized using the Leica TCS 4Pi microscope. Notably, when mounting MAA- and pFA-fixed slides in 97\% TDE as described [Staudt et al., 2007], Qdot655 did not emit fluorescence immediately, but the region of interest had to be excited for at least a minute, before fluorescence would gradually increase to a stable maximum. Since RhodamineX and Qdot655, which exhibit distinctly different spectral properties, were both shown to be equally suitable for $4 \mathrm{Pi}$ microscopy, we are confident that at least dual-color FISH experiments can be imaged using this microscopic setup. This approach will for the first time allow super-resolved 3D distance measurements between specific DNA sequences in the interphase nucleus by 4 Pi microscopy, for example between 2 differentially labeled gene loci. 


\section{Conclusions}

At present, using confocal microscopy it is technically possible to spectrally discriminate up to 6 conventional fluorochromes in 3D-FISH experiments (DAPI, Alexa488, Alexa514, Cy3, Texas Red and Cy5) [Walter et al., 2006; Cremer et al., 2007; Müller et al., 2007]. Recently, we could further extend this number by the introduction of Cy5.5 as a 7th label (our unpublished results). Here we provided the proof of principle that Qdot conjugates can be combined with traditional organic fluorochromes for the visualization of chromosome-painting probes in FISH experiments. At present, at least 2 of the Qdot conjugates meet the requirements both for the analysis of metaphase chromosomes and 3D-preserved interphase nuclei. This new development would further increase the number of spectrally discernable DNA probes in 3DFISH experiments to 8. Although we have not demonstrated this in the present work, we have no reason to believe that our proposed protocol is limited to FISH with whole chromosome paint probes, because successful Qdot detection of locus-specific genomic BAC probes, repetitive sequences and even oligo-nucleotide probes has been previously reported [Pathak et al., 2001; Xiao and Barker, 2004; Knoll, 2007]. For the future, we anticipate rapid progress in the manufacturing procedures of quantum dots, which will further enhance their reliability and robustness in the various fields of biological applications, including FISH.

This technical development should further enhance our possibilities aiming to establish three-dimensional maps of gene loci, to determine the chromatin conformation within a specific genomic region of an individual chromosome territory, or to identify loci involved in specific spatio-temporal cis or trans interactions. Here, each additional probe that can be simultaneously mapped by FISH further increases the number of obtained 3D distances and 3D angles between loci almost exponentially, and accordingly the resolution of higher-order chromatin topology maps.

Finally, our demonstration that at least the 2 fluorochromes RhodamineX and QdotQ655 are suitable for FISH probe imaging by 2-photon 4Pi microscopy opens several new avenues for future studies on nuclear architecture. For example, this would allow high precision interphase distance measurements between genomic loci, quantification of surface interactions and intermingling between neighboring chromosome territory surfaces, or chromatin texture analyses of chromosome territories, or of well-defined chromosome sub-regions.

\section{Acknowledgements}

We thank Tebu-bio for kindly providing Qdot conjugates. We further acknowledge Leica Microsystems, Mannheim, Germany, for access to the TCS 4Pi laser scanning confocal microscope, and in particular Dr. Tanjef Szellas for his helpfulness in capturing and processing $4 \mathrm{Pi}$ confocal image stacks. M.C. and F.G. were supported by CIPSM.

\section{References}

Alivisatos AP, Gu W, Larabell C: Quantum dots as cellular probes. Annu Rev Biomed Eng 7: 55-76 (2005).

Bolzer A, Kreth G, Solovei I, Koehler D, Saracoglu $\mathrm{K}$, et al: Three-dimensional maps of all chromosomes in human male fibroblast nuclei and prometaphase rosettes. PLoS Biol 3: e157 (2005).

Bruchez MP: Turning all the lights on: quantum dots in cellular assays. Curr Opin Chem Biol 9:533-537 (2005).

Bruchez M, Hotz CZ: Quantum dots. $1^{\text {st }} \mathrm{Ed}(\mathrm{Hu}-$ mana Press, Totowa 2007)

Bruchez M, Moronne M, Gin P, Weiss S, Alivisatos AP: Semiconductor nanocrystals as fluorescent biological labels. Science 281:20132016 (1998).
Byers RJ, Di Vizio D, O’Connell F, Tholouli E, Levenson RM, et al: Semiautomated multiplexed quantum dot-based in situ hybridization and spectral deconvolution. J Mol Diagn 9:20-29 (2007)

Chan WC, Nie S: Quantum dot bioconjugates for ultrasensitive nonisotopic detection. Science 281:2016-2018 (1998).

Chan WC, Maxwell DJ, Gao X, Bailey RE, Han $M$, Nie S: Luminescent quantum dots for multiplexed biological detection and imaging. Curr Opin Biotechnol 13:40-46 (2002).

Cremer M, Müller S, Köhler S, Brero A, Solovei I: Cell preparation and multicolor FISH in 3D preserved cultured mammalian cells. CSH Protocols:doi:10.1101/pdb.prot4723 (2007).
Egner A, Hell SW: Fluorescence microscopy with super-resolved optical sections. Trends Cell Biol 15:207-215 (2005)

Hell S, Stelzer EHK: Properties of a 4 Pi-confocal fluorescence microscope. J Opt Soc Am A 9: 2159-2166 (1992).

Jiang Z, Li R, Todd NW, Stass SA, Jiang F: Detecting genomic aberrations by fluorescence in situ hybridization with quantum dots-labeled probes. J Nanosci Nanotechnol 7: 4254-4259 (2007).

Knoll JH: Human metaphase chromosome FISH using quantum dot conjugates. Methods $\mathrm{Mol}$ Biol 374:55-66 (2007).

Ma L, Wu SM, Huang J, Ding Y, Pang DW, Li L: Fluorescence in situ hybridization (FISH) on maize metaphase chromosomes with quantum dot-labeled DNA conjugates. Chromosoma 117:181-187 (2008). 
Medintz IL, Uyeda HT, Goldman ER, Mattoussi $\mathrm{H}$ : Quantum dot bioconjugates for imaging, labelling and sensing. Nat Mater 4:435-446 (2005).

Müller S, Neusser M, Köhler D, Cremer M: Preparation of complex DNA probe sets for $3 \mathrm{D}$ FISH with up to six different fluorochromes. CSH Protocols:doi:10.1101/pdb.prot 4730 (2007).

Murray CB, Norris DJ, Bawendi MG: Synthesis and characterization of nearly monodisperse $\mathrm{CdE}(\mathrm{E}=\mathrm{S}, \mathrm{Se}, \mathrm{Te})$ semiconductor nanocrystallites. J Am Chem Soc 115:8706-8715 (1993).

Neusser M, Schubel V, Koch A, Cremer T, Müller S: Evolutionarily conserved, cell type and species-specific higher order chromatin arrangements in interphase nuclei of primates. Chromosoma 116:307-320 (2007).
Pathak S, Choi SK, Arnheim N, Thompson ME: Hydroxylated quantum dots as luminescent probes for in situ hybridization. J Am Chem Soc 123:4103-4104 (2001).

Schwarzacher T, Heslop-Harrison, JS: Practical in situ Hybridization (BIOS Scientific Publishers Limited, Oxford 2000).

Staudt T, Lang MC, Medda R, Engelhardt J, Hell SW: 2,2'-thiodiethanol: a new water soluble mounting medium for high resolution optical microscopy. Microsc Res Tech 70:1-9 (2007).

Walter J, Joffe B, Bolzer A, Albiez H, Benedetti PA, et al: Towards many colors in FISH on 3D-preserved interphase nuclei. Cytogenet Genome Res 114:367-378 (2006).
Watson A, Wu X, Bruchez M: Lighting up cells with quantum dots. Biotechniques 34:296300, 302-293 (2003).

Wu SM, Zhao X, Zhang ZL, Xie HY, Tian ZQ, et al: Quantum-dot-labeled DNA probes for fluorescence in situ hybridization (FISH) in the microorganism Escherichia coli. Chemphyschem 7:1062-1067 (2006).

Wu X, Liu H, Liu J, Haley KN, Treadway JA, et al: Immunofluorescent labeling of cancer marker Her2 and other cellular targets with semiconductor quantum dots. Nat Biotechnol 21:41-46 (2003).

Xiao Y, Barker PE: Semiconductor nanocrystal probes for human metaphase chromosomes. Nucleic Acids Res 32:e28 (2004). 